\title{
Spirochaetemia in a HIV Positive Patient
}

\author{
E.E. Okwori MBBS, FMC-Path \\ Department of Pathology, Federal Medical Centre, Makurdi, Nigeria
}

\begin{abstract}
Background: Borreliosis, caused by Borrelia recurrentis and several other Borrelia species is not a commonly reported case in our environment, but the search for the cause of recurrent pyrexia in this patient made it possible to discover the spirochete as the cause of the disease.

Method: A 38 year old married HIV positive woman presented with recurrent fever in a private clinic. Six thin smears were made out of the patient serum and dried in the air. Three slides each were stained with $0.12 \%$ Leishman and 20\% Giemsa stains and examined under the light microscope.
\end{abstract}

Result: Three of the Giemsa slides were positive for spirochetes (4-5 spirals), which were constituents with Borrelia species. The patient responded very well to tetracycline and serum became negative for the organism after ten days of treatment.

Conclusion: Borrelia was discovered to be the cause of the recurrent pyrexia in this patient who responded very well to tetracycline. Borrelia should be looked for in cases of pyrexia of unknown origin

KEYWORDS: Borrelia; Human immunodeficiency virus; Tetracycline.

Paper accepted for publication

\section{INTRODUCTION}

Relapsing fever a borreliosis is a human disease, caused by Borrelia recurrentis and several other Borrelia species ${ }^{1,2}$. These bacteria appear as helical rods with about 3-10 loose spirals.'.

Endemic or tick borne relapsing fever is caused by several Borrelia species including Borrelia duttoni, Borrelia lemisii, Borrelia parkeri and Borrelia turicatae. They are transmitted to human by soft-bodied ornithodorus ticks ${ }^{2,3}$.

The cause of epidemic or louse borne relapsing fever is an obligate human pathogen, transmitted from person to person by the body louse, pediculus humanus $^{3,4}$. In both forms of relapsing fever, symptoms include high fever, rigors, headache, myalgia, arthralgia, photophobia and cough. They develop about one week after infection ${ }^{4,5}$. Relapses of fever occur 7-10 days later and several relapses may take place. Relapses is said to be as a result of the antigenic variability of the organism.
In our environment, Borreliosis is not commonly associated with recurrent pyrexia. Common cause of recurrent pyrexia such as malaria and enteric fever were looked for in this patient, but the diagnosis failed. The discovery of this rare condition in our environment needs to be brought to the knowledge of our other colleagues.

\section{CASE REPORT}

A 38 year old married HIV positive woman presented to a private clinic with recurrent fever. The fever had lasted for three months before her presentation. The fever reoccurred every two weeks. She had attended about five clinics before referral to our clinic. She received treatment for malaria and typhoid fever in these clinics, with no improvement.

On examination, she was wasted, body weight of $48 \mathrm{~kg}$. She was mildly pale with PCV of $32 \%$, anicteric and febrile to touch, with temperature of $38^{\circ} \mathrm{C}$.

Laboratory investigations showed no malarial parasite in the blood, and test for salmonelliosis both by culture and immunologically were negative. She was HIV 1 seropositive using two recommended test kits with a CD4 count of 183 cells/ul.

Six thin smears were made from her serum, and three each stained with $0.12 \%$ Leishman and $20 \%$ Giemsa stains. Three of the Giemsa slides were positive for spirochetes (4, 5 spirals) which were consistent with Borrelia species.

The patient was then placed on tetracycline, $500 \mathrm{mg}$ @ six hourly for ten days. The patient responded very well to the tetracycline and fever disappeared and the blood smears became negative to the organism after ten days of treatment.

\section{DISCUSSION}

Borreliosis a human disease, spread by the bite of the human body louse ${ }^{8}$. Other species can be spread by rodents, which serve as a reservoir for the infection. The recurrent nature of the fever they cause and their virulence is due to the organism's ability to undergo antigenic variation ${ }^{8,9}$. Thus as the host mounts an immune response against one antigenic type, another type appears. This effectively nullifies the body's ability to clear the organisms ${ }^{9,10}$.

The recurring bouts of fever correspond to the emergence of new antigenic types ${ }^{10}$. The antigenic 
variation occurs as a result of a genetic rearrangement where silent genes are moved to an expression locus ${ }^{10}$.

Pyrexia of unknown origin (PUO), recurrent fever and relapsing fever are common presentations, especially among people living with HIVIAIDS, because of their low immunity. Association of Borreliosis with them is not very common in Nigeria. This may be because it is not often looked for.

For this particular patient the organism was looked for because the search for common organisms such as malaria, other bacterial infections and viral hepatitis did not yield positive result.

It is therefore recommended that borrelia species should be looked for in all cases of recurrent fever and pyrexia of unknown origin especially as the procedure is cheap and easy to perform.

\section{REFERENCS}

1. Barbour AG.Antigenic variation of Borrelia species. Annual Review of Microbiology 1990; 44: 155-171.

2. Barbour AG, Hayes SF. Biology of Borrelia species. Microbiological Review 1986; 50: 381-400.
3. Guy EC. The Laboratory diagnosis borreliosis. Review in Medical Microbiology 1993; 4: 89-96.

4. Holt SC. Anatomy and chemistry of spirochetes. Microbiological Review 1978; 42: 114-160.

5. Szezepanski A, Benach JL. Lyme Borreliosis: host responses to borrelia burgdorferi. Microbiological Review 1991; 55: 21-34.

6. SteereAG. Lyme disease. New Engl J of Med 1989; 321: 303-308.

7. O Connel, S.Lyme disease in United Kingdom. BMJ 1995; 310: 303-308.

8. Casjens L, Sherwood HA. Bacterial genome in flux: the twelve linear and nine circular extrachromatomal DNAs in an infection isolate of the Lyme disease spirochete Borrelia Burdorferi. Molecular Microbiology 2000; 35:490-516.

9. Eggers E, Christian H. Transduction by PhiBB-1, a bacteriophage of Borrelia Burdorferi. American Society for Microbiology 2001; 183: 4771-4778.

10. Pichon B, Roggers M, Egan D, Gray J. "Blood-meal analysis for the identification of reservoir hosts of tick borne pathogens in Ireland" Vector Borne Zoonotic Dis 2005; 5(2): 172-180. 